\title{
LASER DOPPLER VELOCIMETER MEASUREMENTS IN A
} 3-D IMPINGING TWIN-JET FOUNTAIN FLOW*

\author{
K. R. Saripalli** \\ McDonnell Douglas Research Laboratories \\ St. Louis, MO 63166
}

\section{Abstract}

Mean velocity and turbulence measurements were conducted on the three-dimensional fountain flowfield generated by the impingement of two axisymmetric jets on a ground plane with application to vertical-take-off and landing (VTOL) aircraft. The basic instantaneous velocity data were obtained using a two-component laser Doppler velocimeter in a plane connecting the nozzle centerlines at differcnt heights above the ground emphasizing the jet impingement region and the fountain upwash region formed by the collision of the wall jets. The distributions of mean velocity components and turbulence quantities, including the turbulence intensity and the Reynolds shear stress, were derived from the basic velocity data. Detailed studies of the characteristics of the fountain revealed self-similarity in the mean velocity and turbulence profiles across the fountain. The spread and mean velocity decay characteristics of the fountain were established. Turbulence intensities of the order of $50 \%$ were observed in the fountain.

\section{Nomenclature}

Exit internal diameter of the nozzle or the exit jet diameter

$\mathrm{H} \quad$ Height of the nozzle exit above the ground plate

Re Reynolds number based on the exit jet diameter and the exit jet velocity Center distance between the jets

Mean velocity in the streamwise direction Mean velocity in the cross-stream direction

Fluctuating component of the mean velocity in the streamise direction

$v \quad$ Fluctuating component of the mean velocity in the cross-stream direction

$U_{J} \quad J e t$ centerline velocity at the exit

$U_{\max }^{J}$ The local maximum streamwise velocity in the fountain

$X_{1 / 2}$ The countain half width where $U=\frac{1}{2} U$

$x^{1 / 2} \quad$ Distance parallel to the line connecting the nozzle centerlines

$Y$ Distance perpendicular to the ine connecting the nozzle centerlines and parallel to the ground plane

$\mathrm{Z} \quad$ Perpendicular distance from the ground plane
Introduction

The impinging lift jets of a vertical take-off and landing (VTOL) airoraft hovering in ground proximity produce complicated three-di, 'nsional flowfields. These 3-D flow'ields shown schematically in Fig. 1 for the case of two-iats, involve strong interactions between the impinging lift-jet streams, the airframe surface and the ground. An important feature of this flowfield is the fountain upwash flow generated by the colliding wall jets. The fountain is fan-shaped (Fig. 1), spreading radially in all directions with increasing width away from the ground. The impingement of the fountain on the aircraft increases lift, elevates skin temperatures and causes possible reingestion into the inlets. Detailed studies of the structure and development of the fountain upwash flow are thus essential for the successful design of efficient VTOL aircraft. Toward this objective, a unique set of velocity and turbulence data on an axisymmetric twin-jet fountain flow are presented.

\section{Background}

References $1-4$ report some of the earlier measurements of fountain upwash flow properties. Reference 1 reports mean velocity measurements in

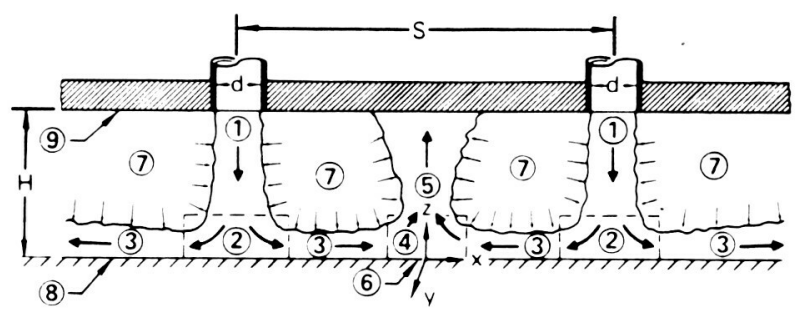

1. Lift jet flow

2. Jet impingement region

3. Wall jet flow

4. Fountain formation region

5. Fountain up-wash flow

6. Wall jet interaction stagnation line

7. Entrainment

8. Ground plane

9. Blocking surface

GP21-1126-3

Fig. 1 Schematic illustration of twin-jet impingement flow.

* This research was conducted under the "connnell Douglas Independent

Research and Development program.

**Scientist; Member A IAA. 
the wall jet and the fountain for twin axisymmetric jet impingement using hot-wire techniques for $S / D=$ 12 and $H / D=5$. The fountain formation and development characteristics were obtained for a range of the important parameters that influence the flow. The flow angularity in the fountain in the plane connecting the nozzle centerlines was reported to vary randomly. Reference 2 presents pitot pressure profiles in the fountain for twin-axisymmetric jet impingement with $S / D=6$ and $2<H / D<5$. Strong fluctuations were reported in the upwash flow properties, and the magnitude of these fluctuations increased with jet height above the ground. Also, highly fluctuating flow angularity, with frequent complete velocity reversals, was reported in the regions outside of the main upwash fan and the shear layers of the incident jets, and sometimes even in the central upwash region. Therefore, it was recommended that pitot probes be used in the measurement of mean flow properties in the fountain upwash.

Reference 3 reported measurements in a twodimensional fountain upwash, indicating high turbulence levels and spreading rates in the fountain. The first set of careful measurements conducted in a two-dimensional fountain upwash using $x$-wire hot film anemometer were presented by Gilbert, who reported detailed mean velocity and turbulence measurements, including decay and spread characteristics of the fountain. The observed levels of turbulent intensities in the fountain were similar to those in an ordinary two-dimensional free jet; however, high growth rates were observed in the fountain. The fountains in References 3 and 4 were generated by two isolated, two-dimensional opposing wall jets, thus eliminating the jet impingement region as part of the fountain formation process.

Reference 5 presents mean velocity and turbulence measurements using hot-wire techniques in a fountain generated by the impingement of two-axisymmetric jets, with no definite conclusions on the fountain turbulence structure. References 6 and 7 describe fountain behavior for close nozzle spacing and also indicate the effect of the blockage by the probe support. Measurements reported in Refs. 8-11 do not reveal any additional features of fountain flows.

Although several investigations of the fountain flow have been carried out, interpretations of the measurements vary widely, primarily because of the difficulty in measuring highly unsteady flows using hot-film and pitot-probe techniques. Computational codes require a better definition of the fountain flow and its turbulence structure to make reliable predictions. Therefore, a need existed for a reliable, detailed mean-velocity and turbulence data base in a realistic 3-D fountain generated by the impinging jets using a suitable measurement technique.

\section{Measurement Technique and the Working Medium}

The measurement technique for studying the fountain flow was required to meet the following specifications: a) be nonintrusive so that no flow disturbance is caused, especially in the case of closely spaced jets, b) be able to sense the direction of flow in the recirculating regions, c) have a linear response, essential for accurate measurements in areas where the turbulence intensities are high such as the fountain. A laser Doppler, yelocimeter (LDV) meets these specifications ${ }^{2,13}$; in addition, LDV responds to a specific velocity component in 3-D flows and measures velocity directly without the need to correct for temperature effects.

As a working medium, water offers specific advantages compared to air; the tracers suitable for flow visualization in water are more numerous with better light-reflecting characteristics, and aerodynamic phenomena can be observed at a relatively slow speed for the same Reynolds number and model scale because of the difference in kinematic viscosities of air and water. Moreover, for laser Doppler velocimetry, the seeding of the flow is normally unnecessary in water because the natural suspended particles act as 1 ight scatterers. If seeding is necessary, it can be accomplished easily in water than in air by adding neutral-density plastic particles of the proper size.

\section{Experimental Apparatus}

Figure 2 shows the jet-impingement facility used for conducting laser-Doppler-velocimeter (LDV) measurements with water as the working medium. The

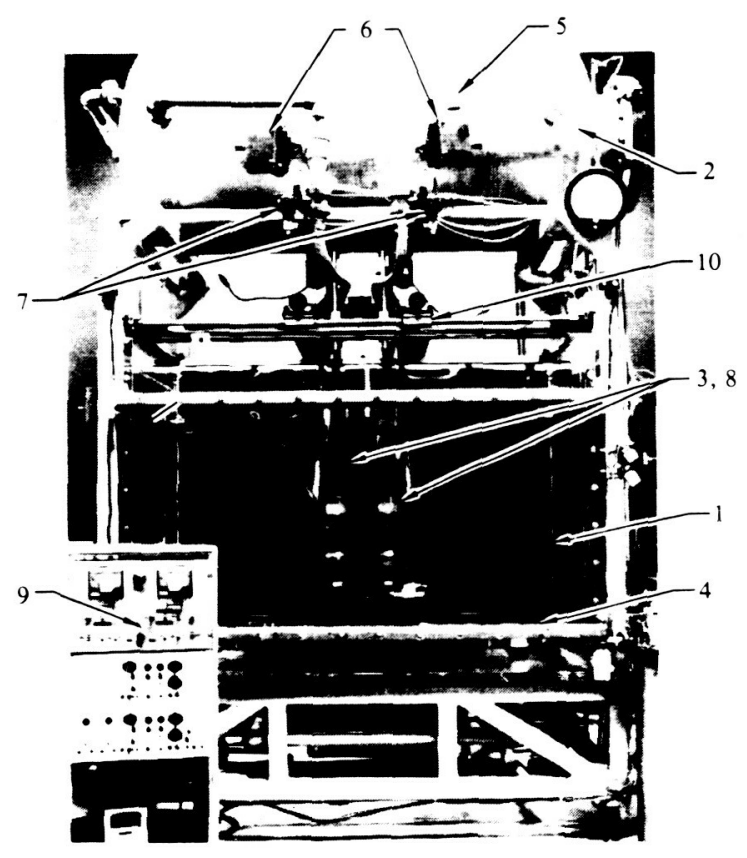

1 Main plexiglass tank

2 Header tank

3 Nozzle units

4 Ground plate

5 Turbine flow meter

6 Motor-operated

flow control valves

7 Shut-off valves

8 Settling chamber of 3

9 Control panet

10 Traversing mechanism

Fig. 2 Jet impingement facility. 
primary components are (1) the main Plexiglas tank, (2) the header tank, (3) nozzle units, and (4) the ground plate. The large transparent main tank $(1.88 \times 1.52 \times 1.37 \mathrm{~m})$ has an approximate capacity of 3800 liters. The pressurized stainless steel cylindrical header tank, $91.4-\mathrm{cm}$ diam., supplies water to the nozzle units and acts as a plenum to damp fluctuations produced by the pump.

The function of the Plexiglas nozzle units (Fig. 3) is to reduce turbulence and mean-velocity nonuniformities in the existing jet-flow to acceptable levels. Each nozzle unit consists of a flow distributor (diffuser) for decelerating the flow, a honeycomb and screens to establish a uniform flow with low turbulence, and a nozzle $(2.54-\mathrm{cm}$ exit diam., 16:1 contraction ratio) for accelerating the flow. The ground plate is held at a fixed height above the bottom of the main tank and acts as an impingement surface for the jets with sufficient edge clearance for passage of the flow. The position of the nozzle units is adjusted by a traversing unit that can accommodate as many as four nozzle units and mounted on the main tank.

The circulation system draws of $f$ water from under the ground plate and pumps it into the header tank, thus supplying water to the jets through a series of flow-control devices. With the available pumping capacity, jet Reynolds numbers up to 200000 can be obtained in a typical twin-jet (2.54-om-exit diam.) impingement configuration. The three-dimensional fountain flow is visualized through a series of two-dimensional images. Fluorescein-sodium, a fluorescent dye, is injected into the jet flow, which fluoresces bright yellowishgreen when the desired cross-section is illuminated with a thin $(1 \mathrm{~mm})$ sheet of $488 \mathrm{~nm}$ wavelength 1 ight from an Argon-ion laser.

A two color (two-component) TSI laser Doppler velocimeter system (Fig. 4) is used in the dualbeam off-axis backward scattering mode with Braggcell frequency shifting. The probe volume is positioned at the required location using a remote$1 y$ driven $X-Y-Z$ traversing unit. The TSI counter-

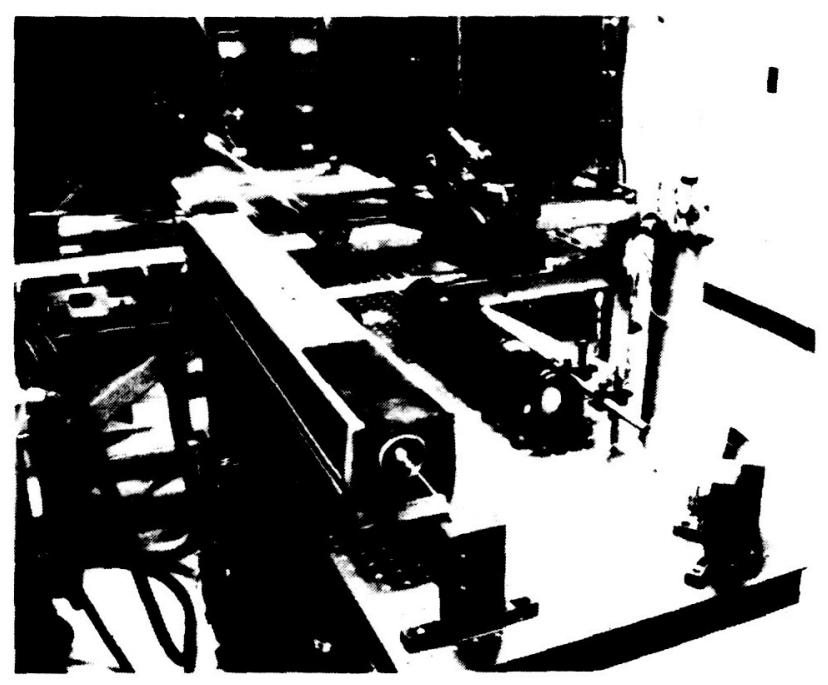

Fig. 3 Laser Doppler velocimeter. type signal processors were used to convert the Doppler signal into a form suitable for recording on a magnetic disk through a dedicated DEC MINC $11 / 23$ Computer. Six groups of 256 samples each were taken at each data point. The recorded data on the disk were then processed on a DEC PDP 11/70 minicomputer and plotted on a Benson-Varian electrostatic plotter. The available city water did not have enough scattering particles to give good signal to-noise ratios in the backward scattering mode; seeding the test medium with $15.5 \mu \mathrm{m}$ Dow Corning polystyrene particles resulted in signals of excellent quality. A TSI hot-film anemometer system with a TSI $1231 \mathrm{w}$ conical hot-film probe was also used for diagnostic measurements of the jet flow at the nozzle exits. A detailed description of the experimental apparatus and the flow visualization and measurement techniques is given in Refs. $14-17$.

\section{Test Conditions}

Extensive diagnostic flow visualization studies were conducted for equal strength jets and for varying heights of the nozzle exit above the ground and separation distances between the nozzles. The objective was to select a twin-jet impingement configuration which has a centrally located, strong isolated fountain rising from the ground plate without interference from the free jets. Based on these flow visualization studies, a normally impinging, equal-strength twin-jet configuration with $S / D=9.0$ and $H / D=3.0$ was selected for detailed LDV measurements. Figure 5 shows the corresponding flowfield visualized in a plane connecting the nozzle centerlines using the fluorescent dye/laser light sheet technique.

LDV measurements were taken for equal-strength jets at twelve heights $(Z / D=0.05$ to 2.94$)$ between the nozzle exits and the ground. The jet exit velocity $\left(U_{J}\right)$ was $6.71 \mathrm{~m} / \mathrm{s}$, resulting in a $R e=$ $1.70 \times 10^{5}$. All measurements were taken in the plane of symmetry connecting the nozzle centerlines. The time-dependent streamwise $(U+u)$ and cross-stream $(V+V)$ velocities in the $X$ and $Z$ directions, respectively, were measured directly by the LDV. The mean velocities (U, V) turbulence intensities $\left(\sqrt{u^{2}} / U, \sqrt{v^{2}} / U\right)$, Reynolds shear stress $\left(-\overline{u v} / U^{2}\right)$, and correlation coefficient $\left(-\overline{u v} / \sqrt{\overline{u^{2}}}\right.$ $\sqrt{v^{2}}$ were derived from the basic velocity data.

\section{Experimental Results}

The experimental data are classifled into three categories, (a) jet-exit flow; establishing the quality of the flow exiting the nozzles (b) jet impingement flow (c) fountain upwash flow.

Jet-Exit Flow Measurements were made in the freejet flow exiting the nozzles to establish uniformity of the mean velocity profile and the core turbulence levels. Both the conical hot-film probe and the LDV were used to obtain a cross check of the data. Further, the hot-film measurements also facilitated spectral analysis of the velocity signals. 


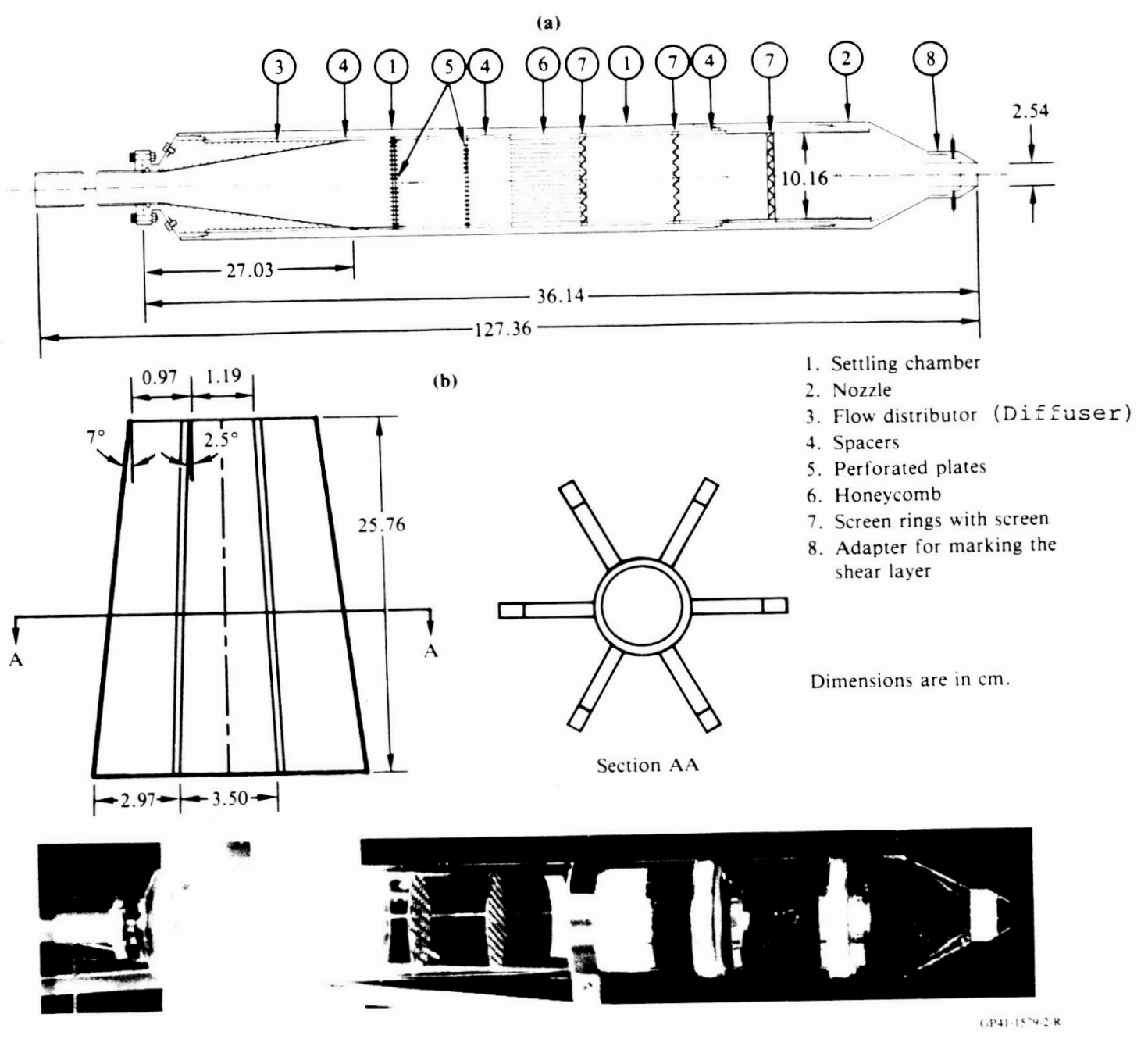

Fig. 4 (a) Schematic of the nozzle unit with the diffuser in place and (b) schematic of the diffuser splitter system.

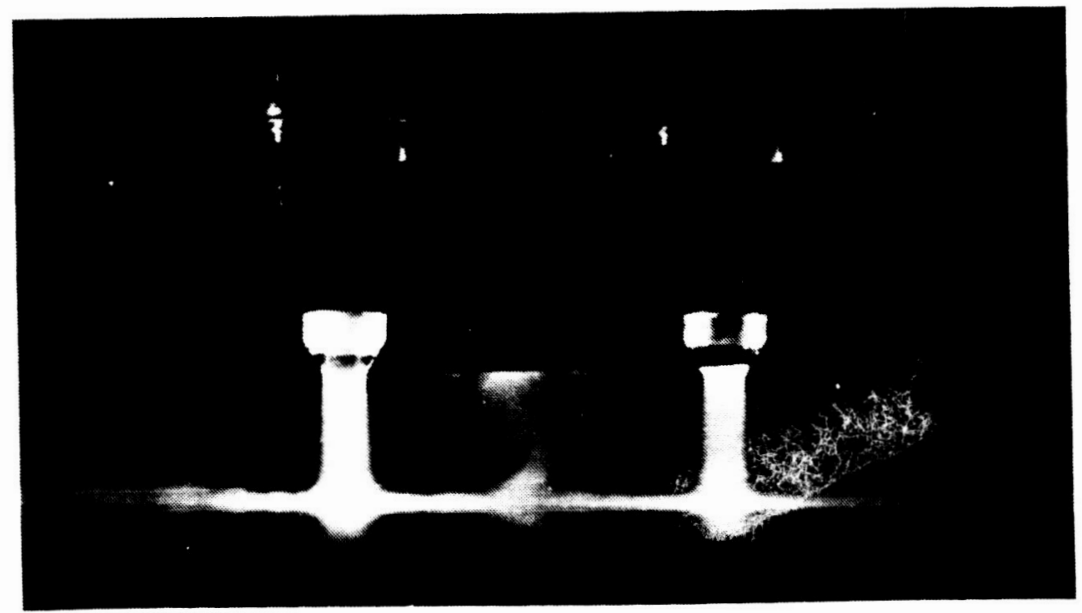

Fig. 5 Twin-jet impingement Plow with fountain

formation: $S / P=9.0, H / D=3.0$, $\operatorname{Re}=1.70 \times 10^{5}$. 
Figure 6 shows the mean-velocity and turbulence-intensity profiles across the jet operating at a $\operatorname{Re}=1.70 \times 10^{5}$, taken $1.5 \mathrm{~mm}$ below the nozzle exit. Here $U$, is the jet centerline velocity at the exit. The jet flow is uniform with a low level of turbulence in the core region; the shear layers are thin. The agreement between the LDV data and hot-film data is good. Because of the large velocity gradients across the probe volume, which was larger than the shear-layer thickness, the LDV gave higher turbulence levels in the shear layers. The frequency spectrum of the hot-film signal taken at the center of the jet showed no selective or specific peaks indicative of unwanted disturbances.

Jet Impingement Flow The mean-velocity and turbulence quantities in the jet were normalized with $\mathrm{U}_{\mathrm{J}}$. Figure 7 shows the streamwise $\left(U / U_{J}\right)$ and cross-stream $\left(V / U_{J}\right)$ components of the mean velocities across the impinging jet at several streamwise locations, with speclal emphasis on the stagnation region. The streamwise $\left(\sqrt{\mathrm{u}^{2}} / U_{J}\right)$ and crossstream $\left(\sqrt{v^{2}} / U_{J}\right)$ turbulence intensities are shown in Figure 8 . The Reynolds shear stress $\left(-\overline{\mathrm{uv}} / \mathrm{U}_{\mathrm{J}}{ }^{2}\right)$
profiles are shown in Figure 9 .

The measurements, especially the $V / U$, data near the ground plate, show a persistent symmetry in the flow about the centerline of the jet. The influence of the ground plate (jet impingement region, Fig. 1) extends to a height of $Z / D=0.75$, where the jet still has a potential core in the streamwise mean velocity profile and the crossstream component of the velocity is close to zero. Below $Z / D=0.75$, the jet starts deflecting along the ground with a rapid decrease in $U$ and increase in $V$. The dip in the $U$ profiles at the center seems typical of jet-impingement flows, as also shown in the data of Donaldson and Snedeker (Ref. 18). Figure 8 shows that in the free jet, away from the ground, $\sqrt{\overline{u^{2}}}=2 \sqrt{\overline{v^{2}}}$; this trend reverses as the jet deflects near the ground. The Reynoldsshear-stress proflles (F1g. 9) near the ground show substantial regions of zero shear stress $(Z / D$.

$0.2)$ despite the large velocity gradients, possibly because the turbulence exhibits a delayed response to the ground plate, as also evidenced by the profiles of $\sqrt{u^{2}}$ and $\sqrt{v^{2}}$ near the ground. Limited measurements on the second jet show simllar initial profiles and jet development.

Fountain Upwash Flow Figure 1011 ustrates the variations of the mean velocities ( $U$ and $V$ ) across the fountain. The distributions of $V$ near the ground reveal that the fountain-formation region (F1g. 1) extends to $Z / D=0.5$, where the variation in $V$ across the fountain is comparable to that at stations farther downstream. However, it should be observed that even at $Z / D=0.2$ and 0.3 , the shape of the $U$ profile resembles that typical for other downstream stations. The turbulence intensities $\sqrt{\overline{u^{2}}}$ and $\sqrt{\overline{v^{2}}}$

ure of the same order of magnitude at a given station above the fountain-formation region $Z / D>0.5$. In the fountain-formation region $2 / D$.< $0.5, \sqrt{u^{2}}$ 5eas observed to be relatively small because of the stagnating flow. The symmetry in the data distributions about the centerline of the fountain reflects the quality of the present measurements. The symmetry in the fountain data was a major problem in the majority of the earlier investigations.

Simllarity of the Fountain Measurements Observation of the mean velocity (Fig. 10) and turbulence profiles in the fountain suggested self-similarity.

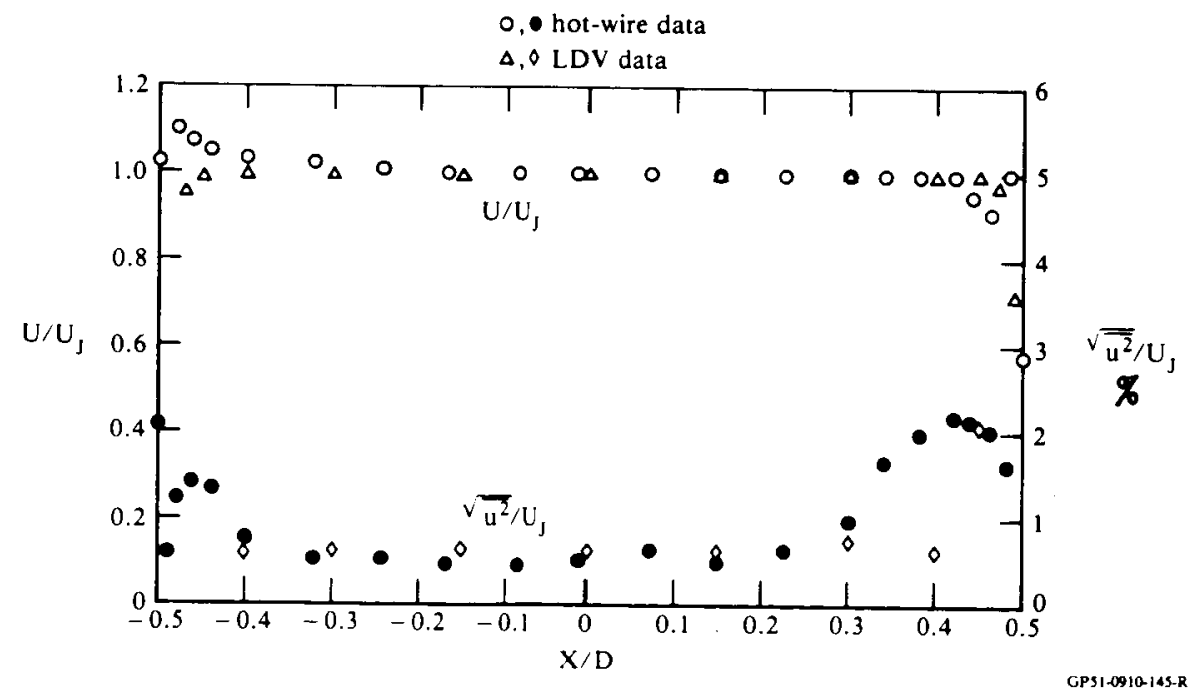

F1g. 6 Mean velocity (U/U $\left.U_{\text {max }}\right)$ and turbulenceintensity $\left(\sqrt{u^{2}} / U_{J}\right)$ proflles at the jet
exit. 


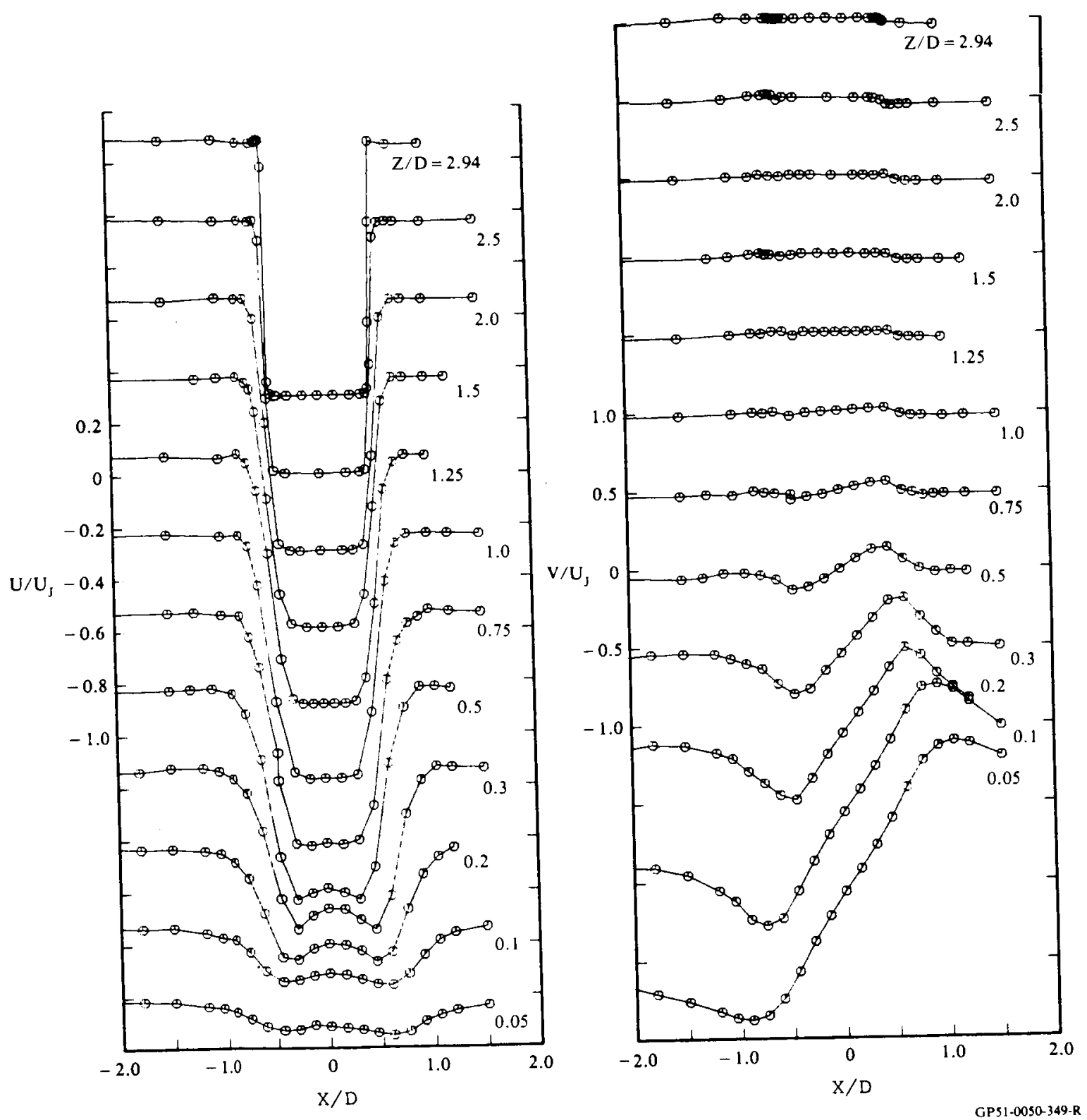

Fig. 7 Variation of (a) streamwise and

(b) cross-stream mean velocities across

the impinging jet. 

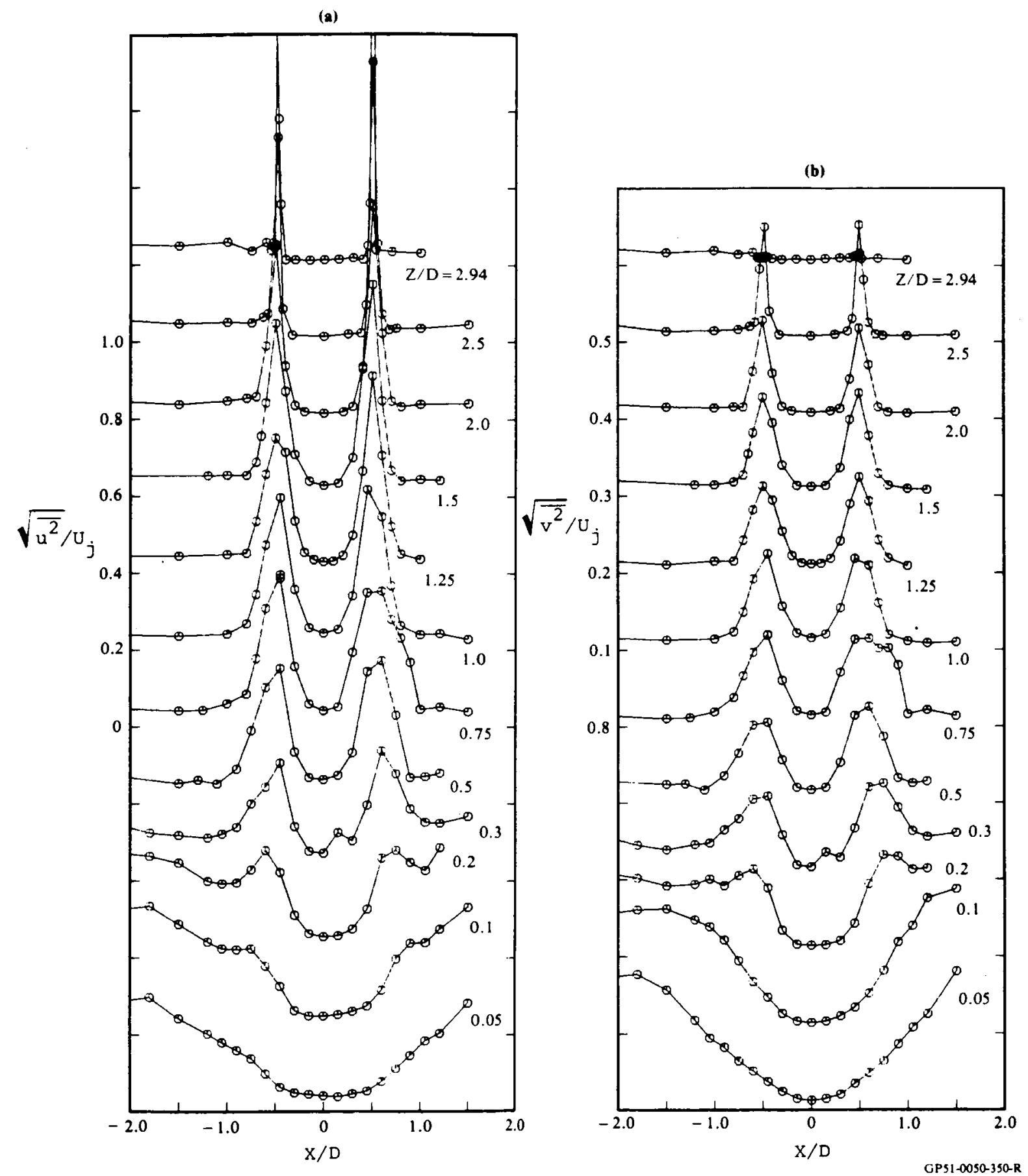

Fig. 8 Variation of (a) streamwise and

(b) cross-stream turbulence intensities across the impinging jet. 


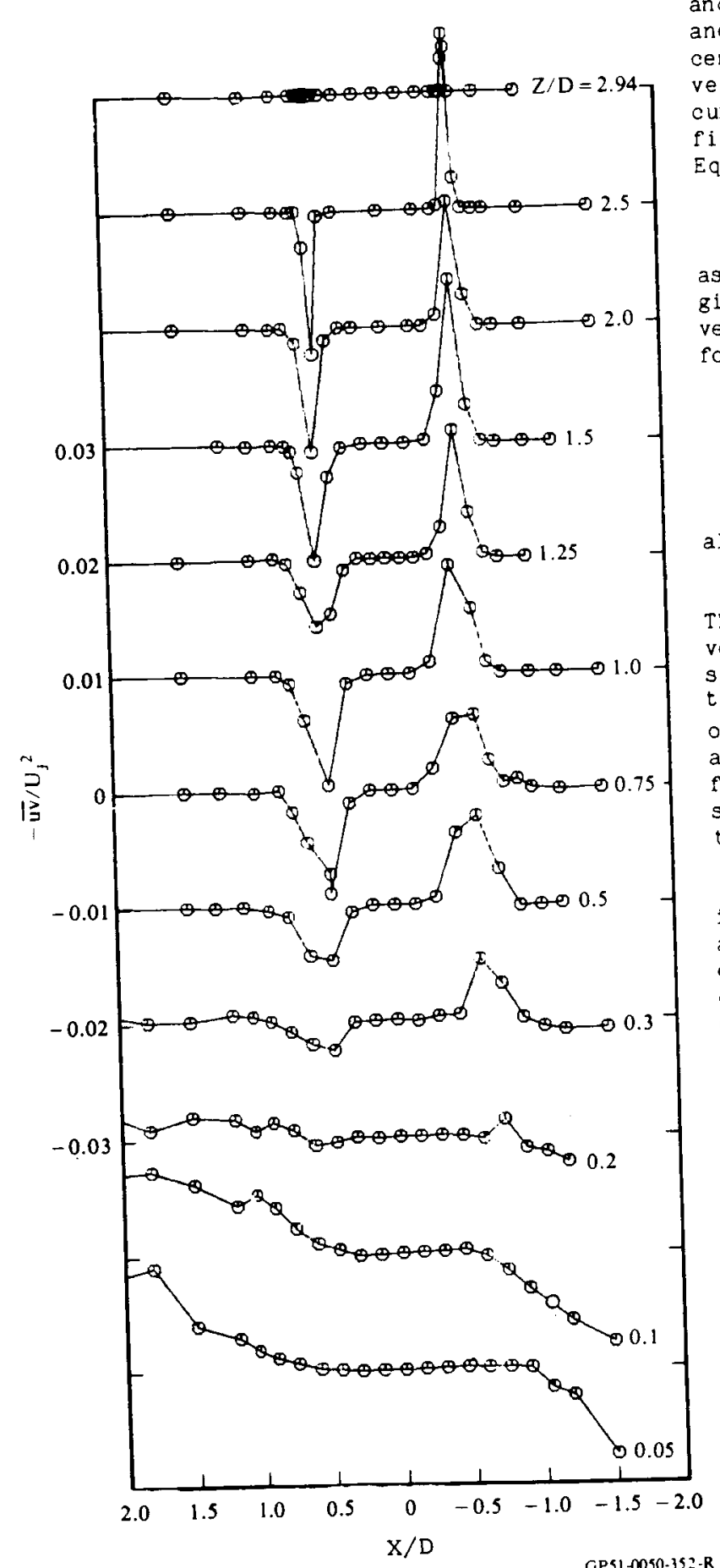

F18. 9 Variation of the Reynolds shear stress across the impinging jet.
The fountain profiles were normalized with $U$ and $X$ where $U$ is the local maximum veloclty and $x_{1 / 2}^{1 / 2}$, the hall $x_{\text {width, }}$ is the distance from the center of the fountain to the point where the velocity is $U / 2$. $U$ and $X_{,}$were obtained by curve-fitting the countaln mean velocity (U) profile with a least square curve of the form shown in Equation (1):

$$
U=A+B \exp \left[-\left(x-x_{0}\right)^{2} / 2 s^{2}\right],
$$

as suggested by Gilbert (Ref. 4). This curve fit gives the symmetry coordinate $X$, the maximum velocity $(A+B)$ and the parameter $S$ related to the fountain half width $x_{1 / 2}$ by Equation (2).

$$
\begin{aligned}
x_{1 / 2} & =\left[2 \operatorname{LOC}\left(\frac{2 B}{B-A}\right)\right]^{1 / 2} S \\
& =1.177 \mathrm{~S}(\text { for } B>>A)
\end{aligned}
$$

also

$$
\left.U / U_{\max }=\exp -0.693\left(X / X_{1 / 2}\right)^{2} \text { (for } B>>A\right) \text {. }
$$

The value of the $X$ for $Z / D \geq 0.5$ was found to be very small establishing once again, the inherent symmetricity in the present data. Flgure 11 shows the linear growth of the fountain half-width $\left(X_{1 / 2}\right)$ obtained by curve-fitting. The growth rate of about 0.16 is lower than that observed in Ref. (4) for two-dimensional fountain upwash. Figure 12 shows the decay of the maximum velocity ( $U$ ) in the fountain again obtained by curve fitting.

Figure 13 shows the streamwise fountain velocity (U) profiles snifted to their symmetry point and normalized with respect to $U_{\text {max }}$ and $X_{1 / 2}$ ' obtalned by curve fitting. A strikling similarity can be observed in the velocity profiles. Similar observations were made for two-dimensional fountain in Ref. (4). The profiles below $Z / D=0.5$ were excluded because they fall into the fountain-formation region, although the profiles at $Z / D=0.3$ and 0.2 do not differ much from the similarity form shown in Fig. 13. The profiles reach a similar form within a short distance $(Z / D=0.5)$ above the fountain-formation region, possibly because of fountain-formation enhanced mixing at the base of the fountain.

The variation of the cross stream velocity ig. 14) through the fountain was also found to be self-similar. These cross-stream mean velocity show an expected smooth variation from +ve on one show an expectedinto -ve on the other with zero crossing at the center of the fountain. The values of $V$ at $Z / D=0.5$ are slightly higher compared to the other downstream stations because of proximity to the fountain formation region.

Figures 15-18 show the various turbulence Figures 15-18 the fountain nondimensionalized quantities across the fountaln $U$ and $x$ obwith the similarity variables $U_{\text {max }}$ and $X$ lizarity can tained by curve fitting. Once again simllarity can be observed in the turbulence proflles at various (Figs. 15 and 16) show that they are generally of (Figs. 15 and 16) show that they are The maxim turthe same magnitude and shape. The maximum tur- 
(b)
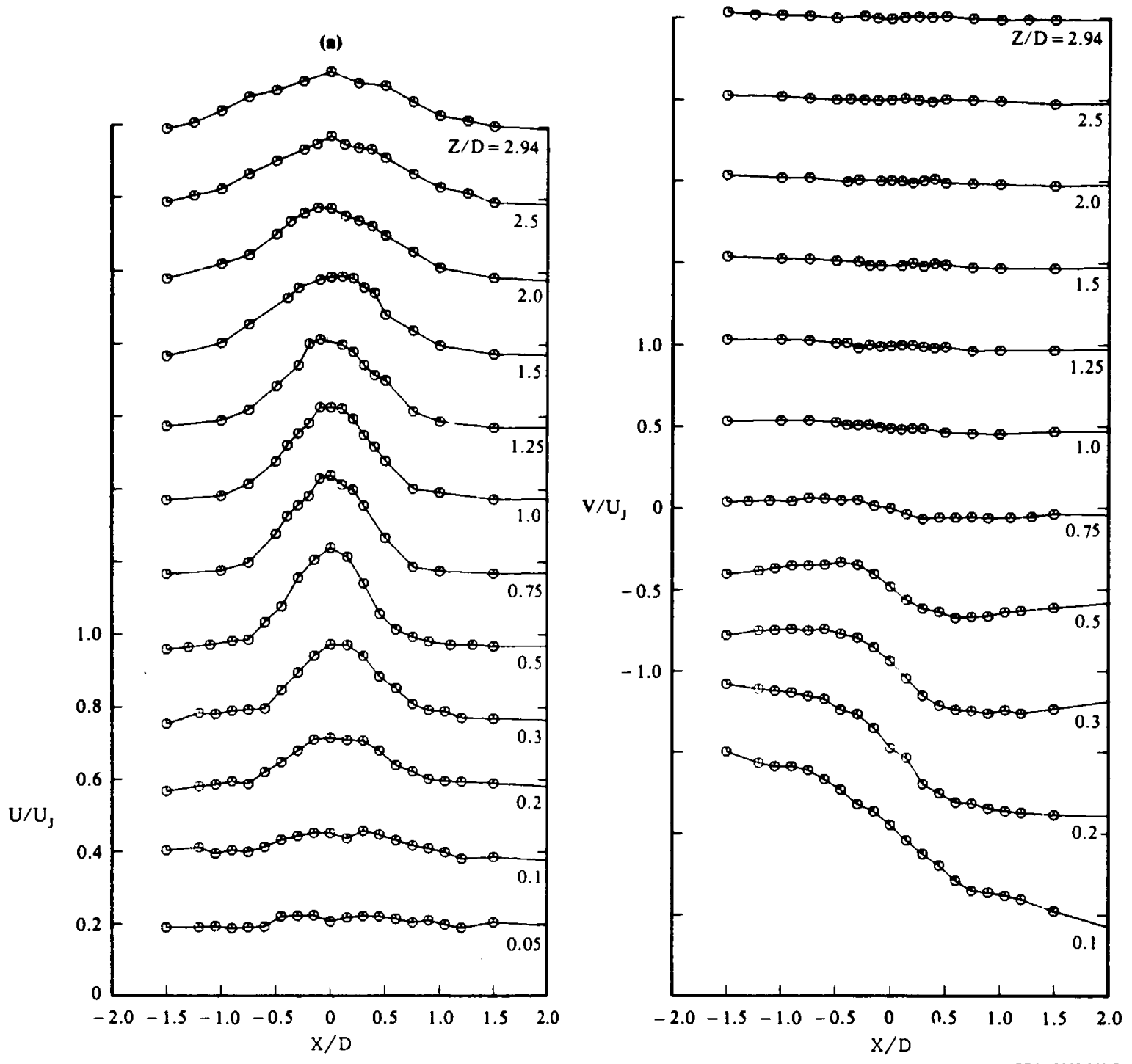

F18. 10 Variation of (a) streamwise and (b) cross stream mean velocities across the fountain.

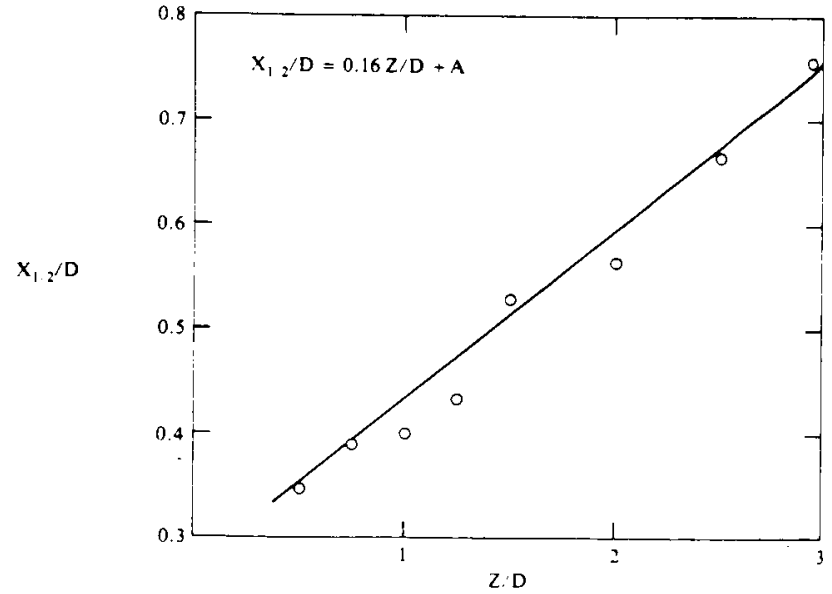

Fig. 11 Growth of the fountain half width $\left(X_{1 / 2}\right)$.

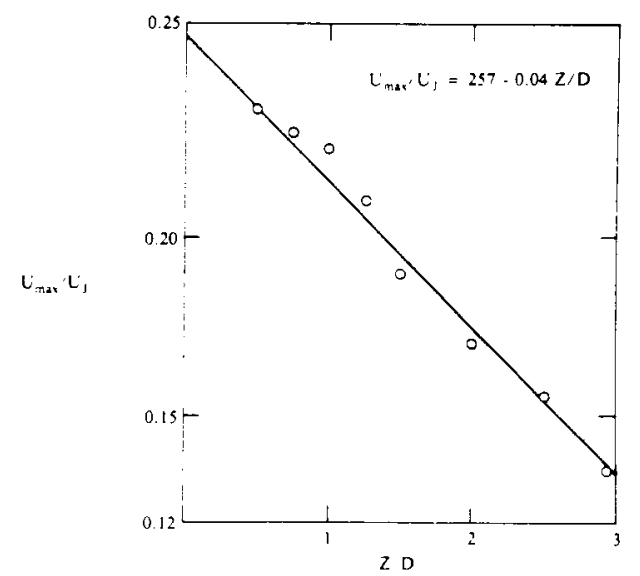

Fig. 12 Decay of the maximum velocity in the fountain (U/ max ). 


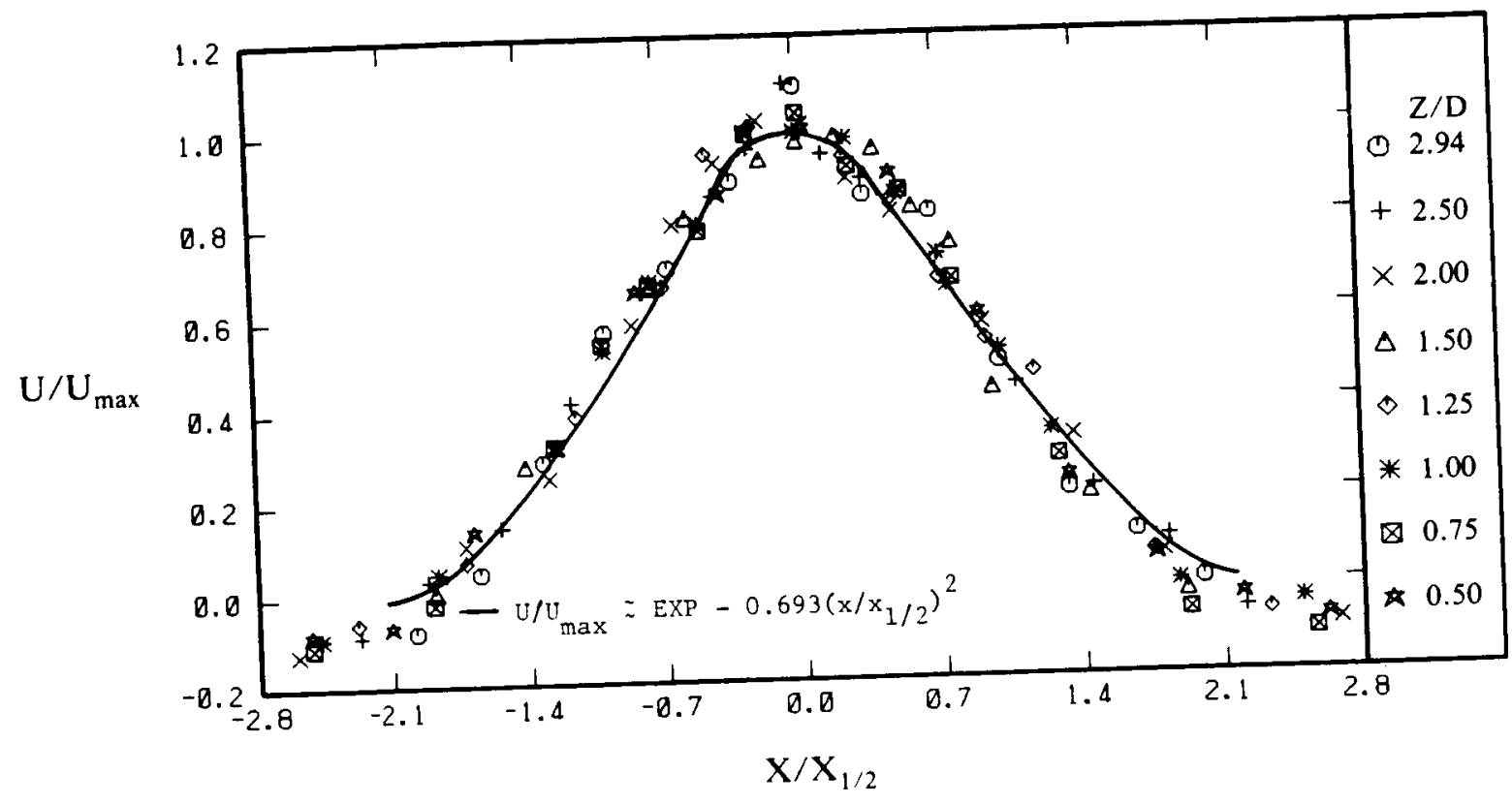

F1g. 13 Similar profiles of streamwise mean

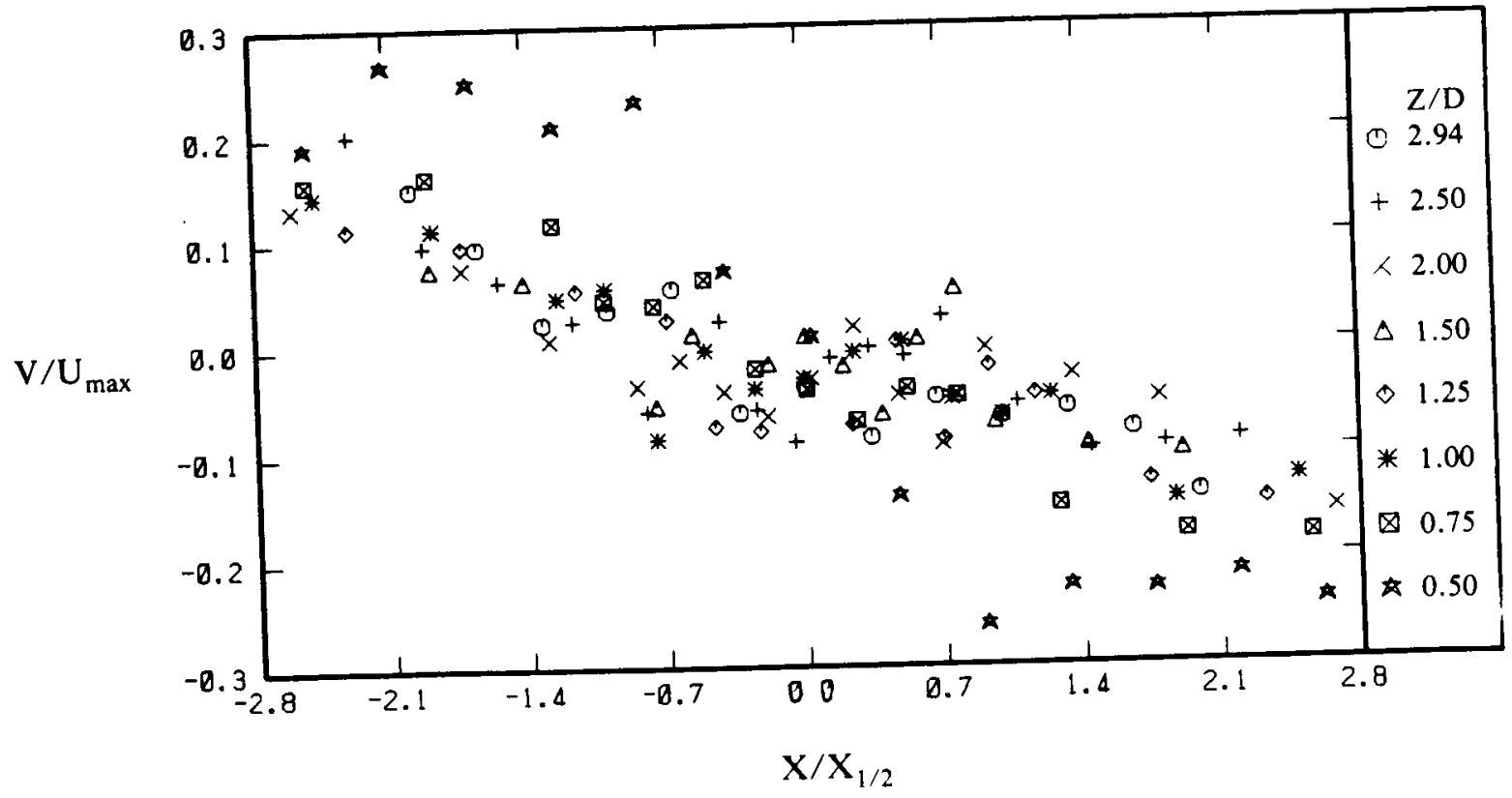

Fig. 14 Similar profiles of cross-stream mean velocity $\left(V / U_{\max }\right)$ across the fountain. 


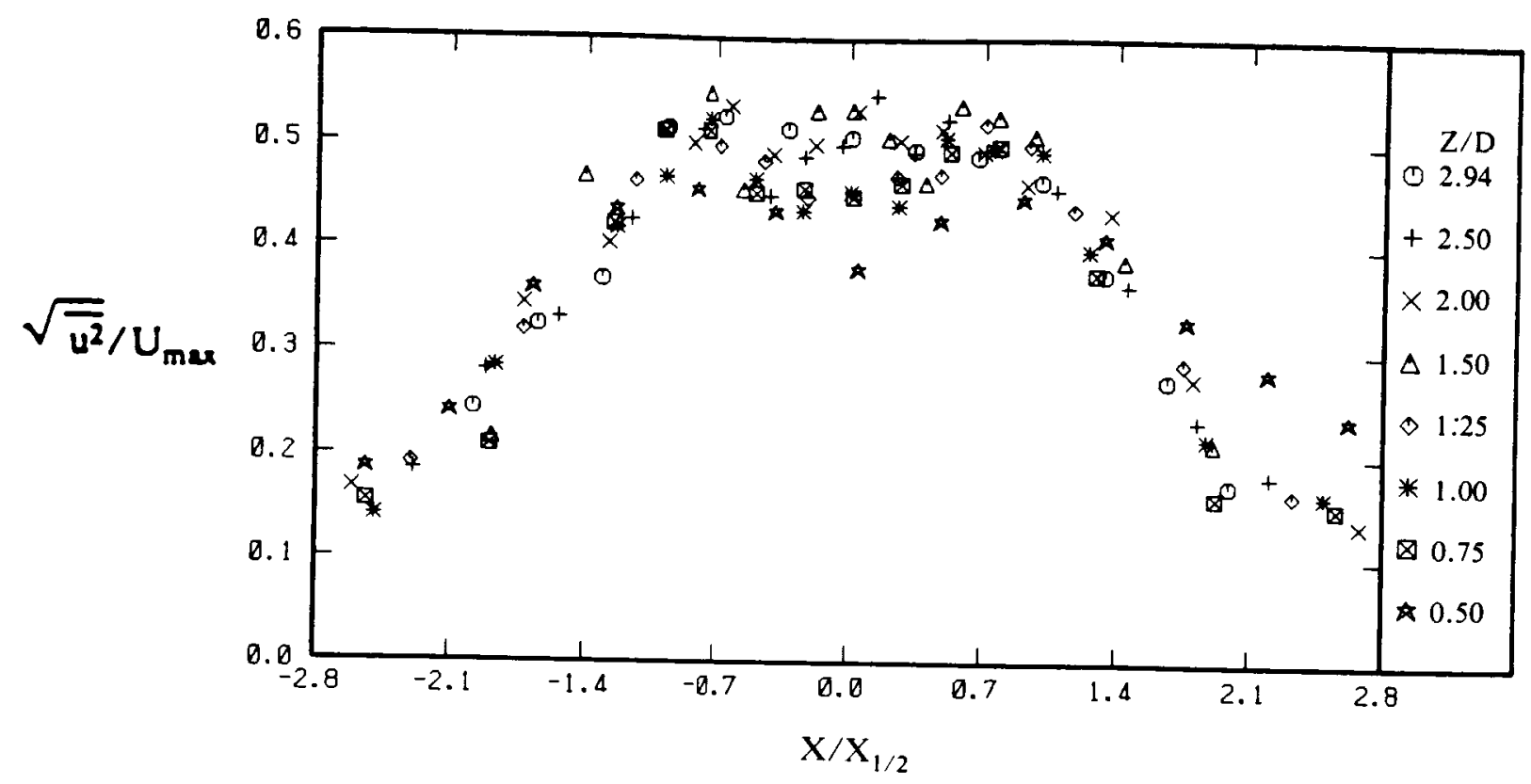

Fig. 15 Similar profiles of streamwise turbulence intensity $\left(\sqrt{\overline{u^{2}}} / U_{\max }\right)$ across the
fountain.

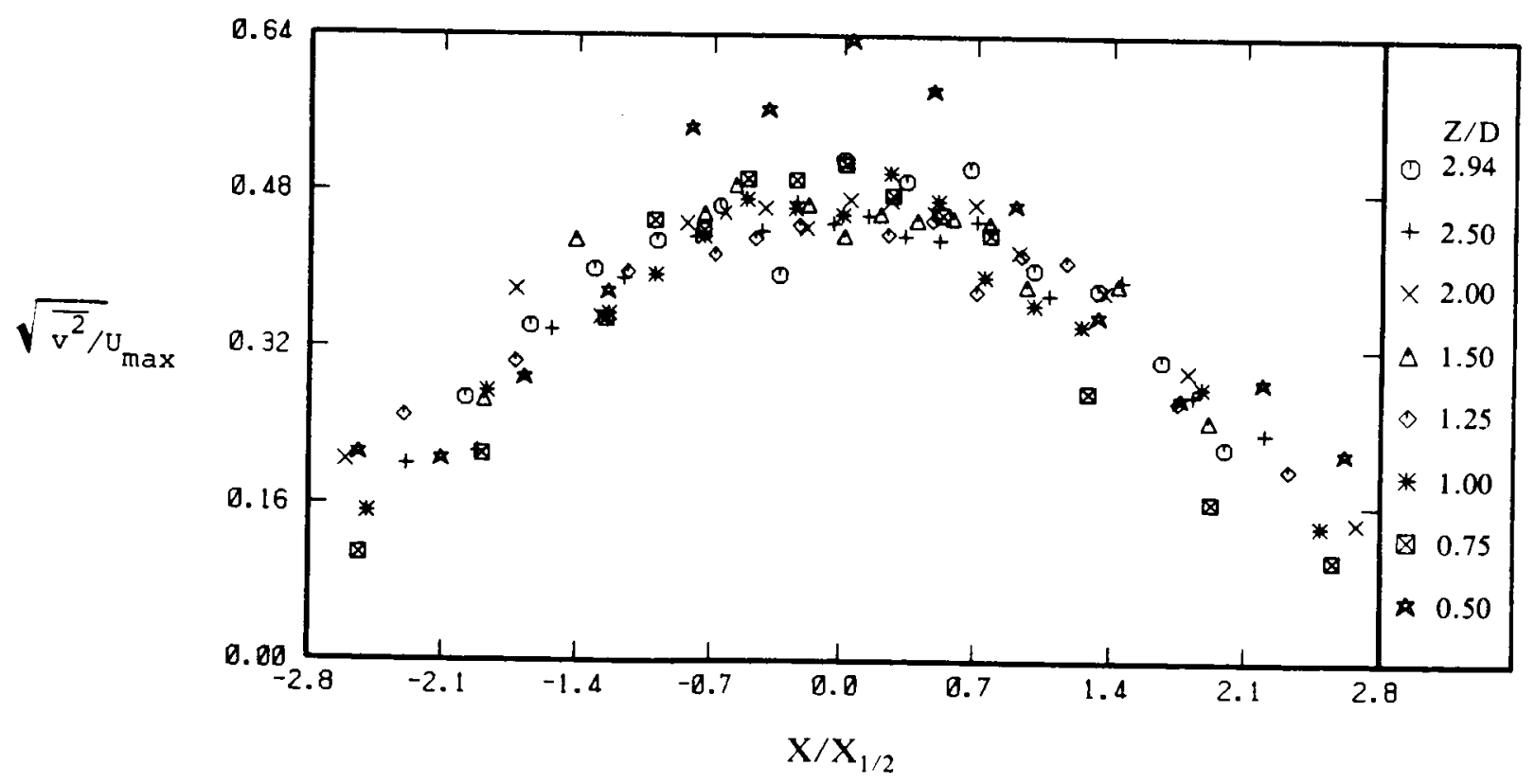

Fig. 16 Similar profiles of cross-stream turbulence intensity $\left(\sqrt{\overline{v^{2}}} / U_{\max }\right)$ across 


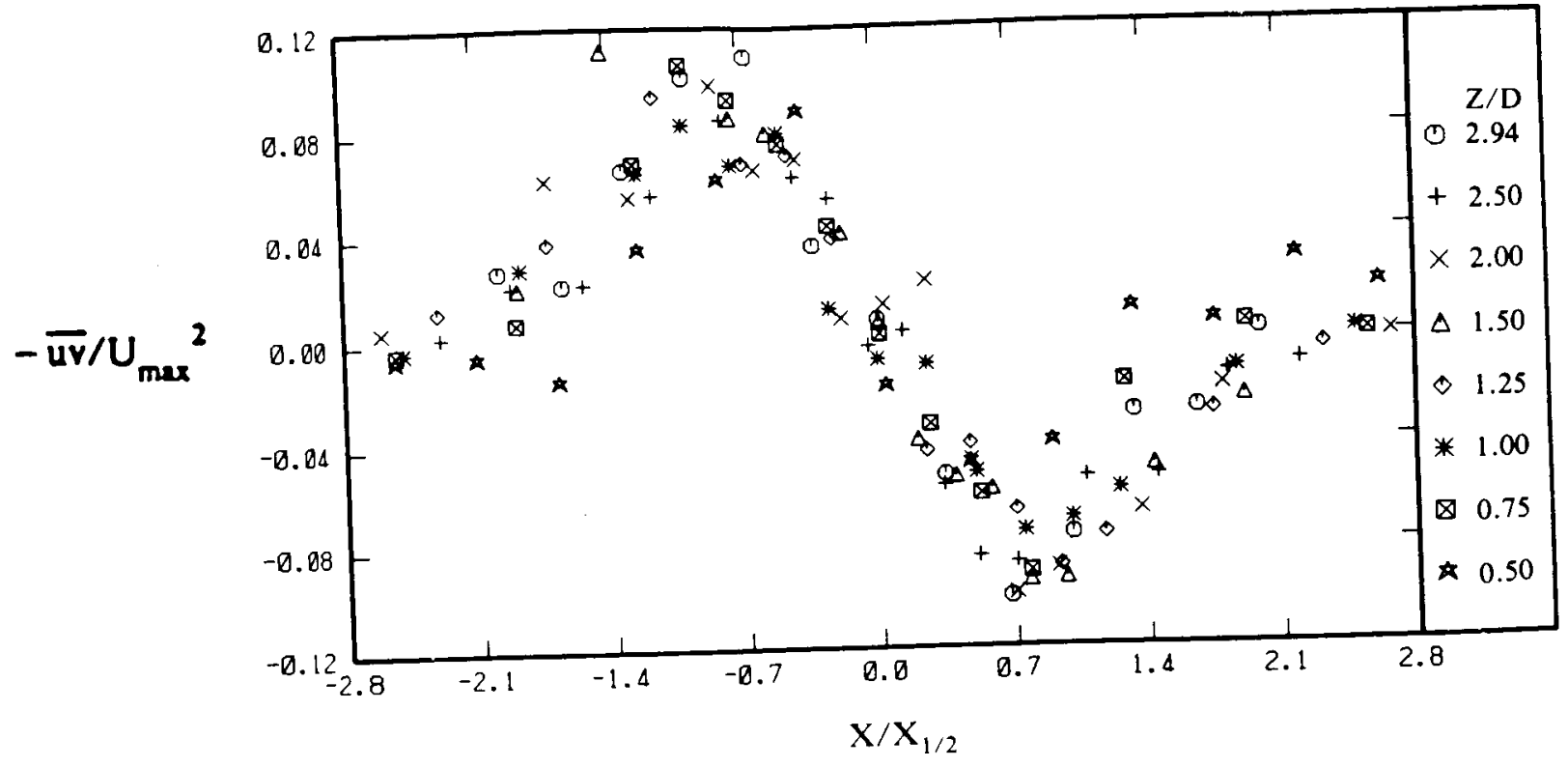

F18. 17 Simllar profiles of Reynolds shear stress $\left(-\overline{u v} / U_{\max }^{2}\right)$ across the fountain.

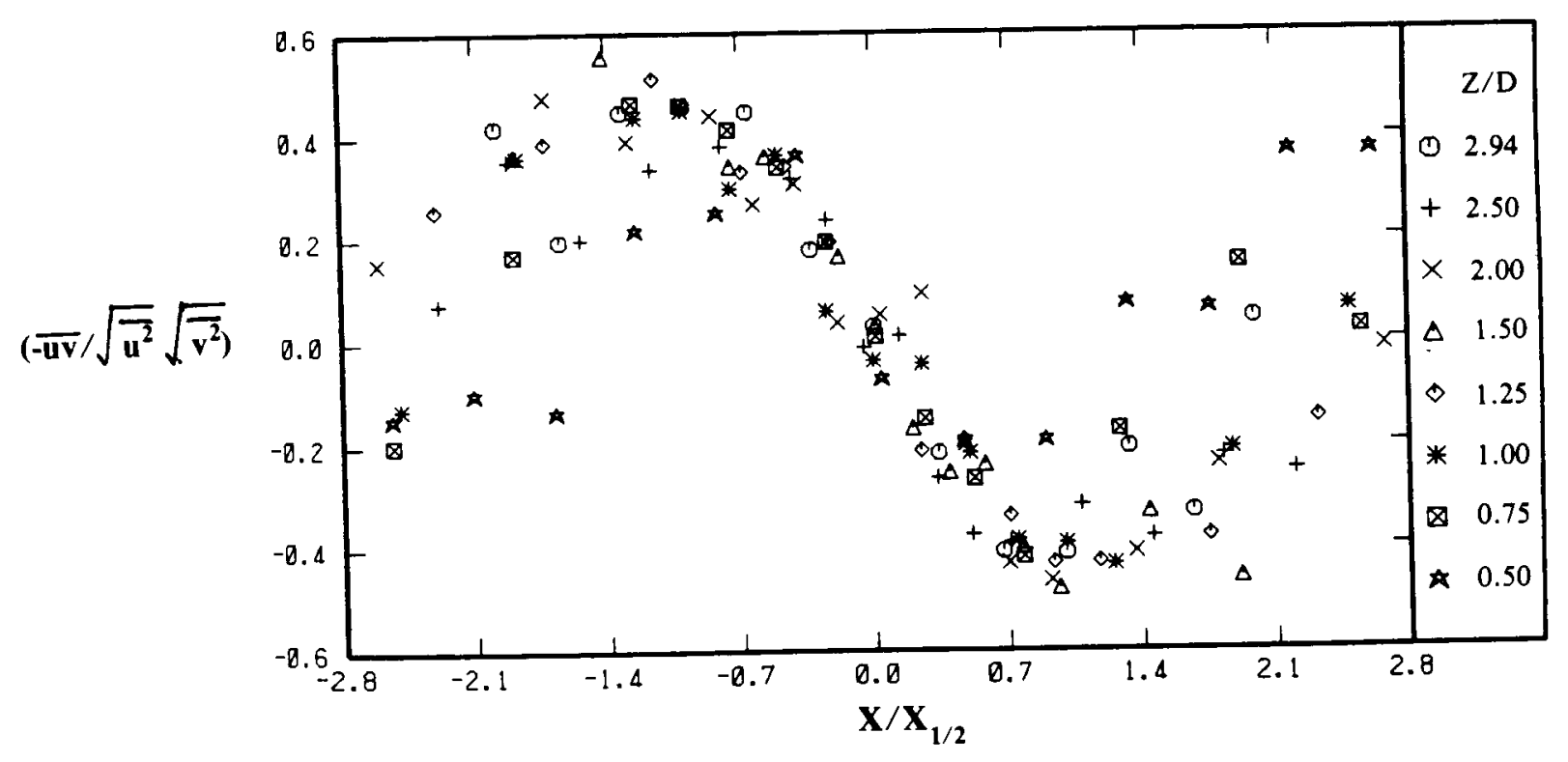

F18. 18 Similar profiles of the correlation function $\left(-\overline{u v} / \sqrt{\overline{u^{2}}} \sqrt{\overline{v^{2}}}\right.$ ) across the fountain. 
local maximum velocity ( $U$ max), suggesting the possibility for even instantaneous flow reversal; therefore the LDV is a more reliable instrument than the hot-film anemometer for measuring fountain flows. The similarity revealed by the Reynolds shear stress (- $\overline{\text { Uv}}$ ) data (Fig. 17) is signiflcant and Indicative of the accuracy and precision of the experiment because the shear stress data in general are particularly sensitive to the measurement techni que.

Figure 18 shows the similarity exhibited by the correlation function $-\overline{u v} / \sqrt{\overline{u^{2}}} \sqrt{\overline{v^{2}}}$. The scatter in the region beyond $\mathrm{X}=\mathrm{X}_{1 / 2}$ is primarily caused by the uncertainty in the measurement of small values of the respective turbulence quantities. The absolute maximum value of the correlation function is observed to be approximately 0.5 .

In general, the turbulence profiles in the fountain were observed to take a longer distance to attaln their self-similar shape than the corresponding mean velocity proflles.

\section{Conclusions and Suggested Future Research}

Mean velocity and turbulence profiles were obtained at 12 stations across the fountain upwash generated by the impingement of two axisymmetric jets using the laser Doppler velocimeter (LDV). Data were also reported on the impinging jet. The turbulence quantities included turbulence intensities, Reynolds shear stresses and correlation functions. The distributions of mean velocity and turbulence quantities across the fountain show self-similarity when nondimensionalized with proper parameters. The fountain flow was observed to be highly turbulent ( $=50 \%$ turbulence intensity) requiring the use of an LDV. The growth of the fountain was observed to be linear at a growth rate of 0.16 . The turbulence and the near ground mean velocity measurements in the three-dimensional fountaln are believed to be the first data reported on such flows.

Additional measurements on the fountain flow away from the symmetry plane connecting the nozzle centerlines using a three component LDV are suggested to fully establish the fountain behavior. The effect of varying the nozzle separation and the helght above the ground on the fountain behavior need to be established.

\section{References}

1. D. R. Kotansky and L. W. Glaze, The Effects of Ground Wall-Jet Characteristics on Fountain Upwash Flow Formation and Development, Report ONR-CR212-216-1F, 15 June 1980.

2. R. C. Jenkins and W. G. Hill, Jr., Investigation of VTOL Upwash Flows Formed by TwO Impinging Jets, Grumman Research Department Report RE-548, November 1977.

3. R. J. Kind and K. Suthanthiran, The Interaction of Two Opposing Plane Turbulent Wall Jets, AIAA Paper 72-211, A IAA 10th Aerospace Sciences Meeting, San Diego, CA, Jan. 1972.

4. B. L. Gilbert, An Investigation of Turbulence Mechanisms in V/STOL Upwash Flow Fields, Grumman Aerospace Rept. Re-688, 1984.
5. W. H. Foley and D. B. Finley, Fountain Jet Turbulence, A IAA Paper No. 81-1293. AIAA Fluid and Plasma Dynamics Conf. Palo Alto, CA, June 1981 .

6. M. J. Siclar1, W. G. H111, Jr., R. C. Jenkins, and D. Migdal, VTOL In-Ground Ef fect Flows for Closely Spaced Jets, AIAA Paper No. 80-1880, August 1980.

7. D. Mlgdal, W. G. Hill, Jr., R. C. Jenkins, and M. J. S1clari, VTOL In-Ground Effect Flows for Closely Spaced Jets, NASA CR-152321, December 1979.

8. G. R. Hall and K. H. Rogers, Recirculation Effects Produced by a Pair of Heated Jets Impinging on a Ground Plane, NASA CR 1307, May 1978.

9. A. Karemaa, C. W. Smith, H. A. Weber, and J. $E$. Garner, The Aerodynamic and Thermod ynamic Characteristics of Fountains and Some FarField Temperature Distributions, Report ONR-CR 212-237-1F, JuIy 1978.

10. J. R. Lummus, The Criticality of Englne Exhaust Simulations on VSTOL Model - Measured Ground Effects, Report ONR-CR212-255-1F, August 1979.

11. M. J. Siclari, W. G. Hill, Jr., and R. C. Jenkins, Investigation of Stagnation Line and Upwash Formation, AIAA Paper No. 77-615, AIAA/NASA, Ames V/STOL Conference, June 1977.

12. F. Durst, A. Melling, and J. H. Whitelaw, Principles and Practice of Laser-Doppler Anemometry, (Academic Press, NY, 1976).

13. T. S. Durrani and C. A. Greated, Laser Systems In Flow Measur ement, (?lenum Press, NY, 1977).

14. K. R. Saripalli and J. C. Kroutil, A Novel Experimental Facility for Conducting JetImpingement Studies Related to VTOL Aircraft, AIAA Paper 85-0052, 1985.

15. K. R. Saripalli, Visualization of Multi-Jet Impingement Flow, AIA A Paper 81-1364, July 1981 .

16. K. R. Saripail1, Visualization Studies of Jet Impingement Flows at MCDonneli Douglas Research Laboratories, Third Intl. Symp. on Flow V1 sualization, Ann Arbor, Sept. 1983.

17. K. R. Saripalli, Visualization of Multi-Jet Impingement Flow, A IAA J. 21, 483 (1983).

18. C. Donaldson and R. S. Snedeker, A Study of Free Jet Impingement. Part I. Mean Properties of Free and Impinging Jets, J. Fluid Mech. 45, 281 (1971). 